\title{
Introduction to the key contents of the Chinese Society of Breast Surgery Practice Guideline 2021
}

\author{
Hongyu Xiang, Qian Liu, Yinhua Liu \\ Breast Disease Center, Peking University First Hospital, Beijing, China \\ Contributions: (I) Conception and design: Y Liu; (II) Administrative support: None; (III) Provision of study materials or patients: H Xiang, Q Liu; (IV) \\ Collection and assembly of data: H Xiang, Q Liu; (V) Data analysis and interpretation: H Xiang, Y Liu; (VI) Manuscript writing: All authors; (VII) \\ Final approval of manuscript: All authors. \\ Correspondence to: Yinhua Liu. Breast Disease Center, Peking University First Hospital, Beijing, China. Email: liuyinhua7520@163.com.
}

\begin{abstract}
Breast disease is one of the most common afflictions for women worldwide. While clinical diagnosis and treatments have progressed rapidly in recent years, surgical procedures are still a mainstay of treatment for breast diseases. The Chinese Society of Breast Surgery (CSBrS) Practice Guideline 2021 which refers to the GRADE (Grading of Recommendations Assessment, Development and Evaluation) system, is due to be published soon. Experts at the CSBrS conducted in-depth discussions on 20 basic issues of breast surgery in China, including the screening of high-risk groups for breast cancer; the diagnosis and treatment of benign and malignant breast diseases; the standard of classic surgical procedures; the surgical techniques used in breast diseases; and clinical genetic testing of breast cancer. Furthermore, due to the absence of high-quality research at present, the expert group gave prudent recommendations on clinical issues such as breast reconstruction surgery and the application of endoscopic technology in the field of breast surgery. The experts prioritized level I and level II evidence into the guideline evaluation system based on the characteristics of clinical research outcomes in China. They also comprehensively considered the accessibility of the Guideline in clinical practice in China, and provided recommendations with different strength. The aim of the Guideline was to provide feasible reference for Chinese breast surgeons in clinical practice.
\end{abstract}

Keywords: Breast surgery; practical guideline; Grading of Recommendations Assessment, Development and Evaluation system (GRADE system); Chinese Society of Breast Surgery (CSBrS)

Received: 11 June 2021; Accepted: 20 July 2021; Published: 31 July 2021.

doi: $10.21037 /$ tbcr-21-8

View this article at: https://dx.doi.org/10.21037/tbcr-21-8

The Chinese Society of Breast Surgery (CSBrS) Practice Guideline 2021 (hereinafter referred to as the Guideline) is soon to be published by the People's Medical Publishing House. This Guideline was compiled by an expert panel in the Chinese Society of Breast Surgery based on the GRADE (Grading of Recommendations Assessment, Development and Evaluation) system. All clinical evidence considered by the Guideline were classified into four categories, namely, I, II, III, and IV, which reflected the reliability of the data quantitatively. In addition, based on the characteristics of the clinical research outcomes in China, the data was prioritized with level I and level II evidence included in the Guideline evaluation system. The CSBrS comprehensively considered the accessibility of the Guideline in clinical practice in China, and provided recommendations with different strengths. The aim of the Guideline was to provide a feasible reference for Chinese breast surgeons in clinical practice.

\section{The norms of basic surgical techniques and operations in patients with breast diseases}

The Globocan 2020 reported 416,000 new cases of breast cancer in China per year (1). In addition, a variety of benign breast diseases seriously affect women's health. The fundamental and clinical research of breast diseases has 
developed rapidly, and the ideas and techniques of breast surgery have also undergone significant changes since the $21^{\text {st }}$ century. However, the geographic condition, level of economic development, and ethnic characteristics of China are different with those of foreign countries. Referring to the international advanced experience of diagnosis and treatment of breast diseases, a practical guideline adapted to China should be explored to improve the medical standards of breast surgery. To achieve this, an expert panel of the $\mathrm{CSBrS}$ conducted in-depth discussions on 20 basic issues of breast surgery in China and compiled the first clinical practice guideline for breast surgery in China. The main contents include the screening of high-risk groups for breast cancer; the diagnosis and treatment of benign and malignant breast diseases; the standard of classic surgical procedures including modified radical mastectomy of breast cancer, breast-conserving surgery, and sentinel lymph node biopsy; and the surgical techniques used in breast diseases including ultrasound-guided breast lesions and lymph nodes biopsy, ultrasound-guided vacuum-assisted breast biopsy (VABB), central venous port implantation, and visualized percutaneous tissue marker clip placement. Clinical genetic testing of breast cancer including multigene profiling assays and BRCA genetic testing are also discussed. Furthermore, due to the absence of high-quality research at present, the expert group gave prudent recommendations on clinical issues such as breast reconstruction surgery and the application of endoscopic technology in the field of breast surgery.

\section{The principle of the guideline: GRADE standard}

GRADE is an evaluation system that grades the quality of the evidence and the recommendation strength of systematic reviews and clinical guidelines (2). The system was first established in 2000. It has been recognized and adopted by numerous international organizations, including the World Health Organization and the American College of Physicians. In the GRADE guideline development process, the clinical problem is first identified and the relevant evidence is collected. The quality of the evidence is then evaluated and the strength of recommendations is assessed. To facilitate this, several committees are established, including the Committee for Evaluation of Evidence, the Literature Research Committee, and the Guideline Writing Committee. To ensure the scientific quality and objectivity of the Guideline, close to 100 specialists from related professions, including breast surgeons, oncologists, radiologists, and statisticians, were involved in the process.

\section{The criteria for the level of evidence}

In accordance with the GRADE standard, the Guideline provided quantified recommendations on the basic principles of diagnosis and treatment of breast diseases by combining the level of evidence with the recommendation strength. The panel of experts referred to the results of international high-quality studies and considered the accessibility of clinical practice in China. The Guideline also included different levels of evidence suitable for Chinese patients (Table 1). To improve the scientific worth and precisions of the Guideline, the experts assigned preference to level I and level II evidence. The Guideline will also be improved as the latest research results become available and will continue to strive to provide Chinese breast surgeons with updated clinical practice references suitable for China.

\section{Criteria for recommendation strength}

The Guideline was originally developed for Chinese breast surgeons, and the accessibility of the Guideline in clinical practice in China should be fully considered. The Guideline made recommendations based on four aspects, namely the level of evidence, health economics, product equivalence, and accessibility. These aspects were considered in conjunction with the GRADE system and characteristics of domestic clinical practice in China. All recommendations were marked according to the weight factor and classified into the following three recommendation strength class: A (strongly recommended), B (weakly recommended), and C (not recommended) (Tables 2,3).

\section{Applicable users of the Guideline and conflicts of interest}

The Guideline was designed for Chinese breast diseases specialists. The expert committee for these guidelines have no conflicts of interest to declare.

\section{Highlights of the guideline}

The Guideline included 20 issues of clinical focus in breast surgery in China, and made recommendations of a variety of strengths according to different levels of evidence and the accessibility of clinical practice in China. 
Table 1 Criteria for the level of evidence

\begin{tabular}{ll}
\hline Level of evidence & \multicolumn{1}{c}{ Criteria } \\
\hline Level I & $\begin{array}{l}\text { Based on high-quality prospective randomized controlled trials, observational studies with large sample } \\
\text { size, meta-analyses, internationally recognized current guidelines and consensus, published guidelines and } \\
\text { consensus from Chinese national colleges or associations, and the above types of studies based on the } \\
\text { Chinese population } \\
\text { Based on low-level randomized trials, well-designed uncontrolled trials, cohort studies, guidelines from } \\
\text { intercontinental associations, consensus of international conferences, published guidelines and consensus } \\
\text { from Chinese regional colleges or associations, and the above types of studies based on the Chinese } \\
\text { population } \\
\text { Based on case-control studies, retrospective studies, guidelines, or consensuses published by national } \\
\text { associations or Chinese provincial colleges or associations, and the above types of studies based on the } \\
\text { Chinese population } \\
\text { Based on case reports, scientific hypotheses, consensus published by different countries or regions, } \\
\text { guidelines and consensus published by Chinese local colleges or associations, and the above type of } \\
\text { studies based on Chinese population }\end{array}$ \\
\hline
\end{tabular}

Table 2 Factors and criteria for recommendation strength

\begin{tabular}{lcl}
\hline Factors & Weight & Criteria \\
\hline Level of evidence & 60 & Level I: 60 points; level II: 45 points; level III: 30 points; level IV: 15 points \\
Health economics & 10 & Consistent with health economics: 10 ; inconsistent with health economical: 0 points \\
Product Equivalence & 10 & Equivalent products or measures: 10 points; no equivalent products or measures: 0 points \\
Accessibility & 20 & Available in China: 20 points; not available: 0 points \\
Total & 100 & \\
\hline
\end{tabular}

Table 3 Recommendation strength

\begin{tabular}{ll}
\hline Recommendation strength & \multicolumn{1}{c}{ Criteria } \\
\hline A & $\begin{array}{l}\text { The average total score of four } \\
\text { factors }>80 \text { points } \\
\text { B }\end{array}$ \\
& $\begin{array}{l}\text { The average total score of four } \\
\text { factors } 60-80 \text { points }\end{array}$ \\
C & The average total score of four \\
& factors $<60$ points \\
\hline
\end{tabular}

\section{Risk assessment of breast cancer and screening of high-risk groups}

To accurately assist physicians with breast cancer risk assessment and screening of high-risk groups in Chinese women, the Guideline gave recommendations on the key clinical issues, emphasizing that breast cancer risk assessment was a preventive measure for healthy women. Due to the lack of high-level evidence for Chinese women at present, clinicians should fully communicate with patients in terms of the purpose of risk assessment, the limitations of current evidence, possible risk reduction methods in the future, as well as the advantages and disadvantages, and provide mental counseling for high-risk groups to reduce their psychological burden. The Guideline recommended online breast cancer risk assessment tools for Chinese women. This recommendation is based on level II evidence and has a class A recommendation strength (3).

\section{Preoperative evaluation of breast cancer}

Before performing operations for breast cancer patients, a comprehensive and standardized preoperative evaluation should be conducted to determine whether there are any factors that may increase the risk of surgery or are detrimental to recovery, as well as potential risks that may affect the entire course of the disease. The Guideline recommended physicians to complete general, anesthetic, and tumor-related assessments for patients before surgery. This recommendation is based on level I evidence and has 
class A recommendation strength (4). Professional genetic counseling was recommended for women with a significant genetic predisposition or family history of breast cancer.

\section{Diagnosis and treatment of benign breast diseases}

The Guideline made recommendations on the diagnosis and treatment of four common benign breast diseases, including hyperplasia of the mammary glands, non-lactation mastitis, breast fibroadenoma, and intraductal papilloma.

Hyperplasia of the mammary glands refers to noninflammatory, non-neoplastic lesions of the breast glands. The Guideline recommended regular follow-up and nondrug therapy as the main treatment. Core needle biopsy (CNB) for patients with indications was recommended as an important method to avoid clinical underestimation (5).

Non-lactation mastitis mainly includes the following two pathological types: periductal mastitis and granulomatous lobular mastitis. The Guideline recommended ultrasound-guided CNB for pathological diagnosis. This recommendation is based on level I evidence and has a class A recommendation strength (6).

Breast fibroadenoma is the most common benign breast tumor in women with a very low risk of carcinogenesis. The Guideline recommended surgical intervention with the following indications: large tumors $>3 \mathrm{~cm}$, rapid growth, increased BI-RADS category, and suggestive atypical hyperplasia or suspected lobular tumor pathologically. Intervention methods include traditional open excision and ultrasound-guided VABB (7).

Intraductal papilloma is prone to recurrence and may be accompanied by pathological changes such as atypical ductal hyperplasia $(\mathrm{ADH})$ and/or carcinoma. Therefore, the Guideline recommended complete resection of the lesion. Open excision or VABB were recommended.

\section{Diagnosis and treatment of malignant breast diseases}

Malignant breast diseases included ductal carcinoma in situ (DCIS) and invasive breast cancer. Pregnancy-associated breast cancer (PABC) requires particular consideration in terms of both maternal efficacy and fetal safety, and thus the diagnosis and treatment of PABC has its own particularities.

DCIS should be diagnosed by postoperative histopathology. Breast-conserving surgery or mastectomy (or combined with breast reconstruction surgery) are the main surgical options. The Guideline recommended that patients diagnosed with DCIS by needle biopsy should undergo sentinel lymph node biopsy (SLNB) simultaneously with breast surgery, avoiding pathological underestimation of breast lesions.

Breast cancer is the most common malignancy in Chinese women. Comprehensive treatment can greatly improve the prognosis of breast cancer patients. The Guideline recommended ultrasound-guided $\mathrm{CNB}$ for breast lesions with BI-RADS 4 or BI-RADS 3 in patients with a family history of breast cancer. This recommendation is based on level I evidence and has class A recommendation strength. Furthermore, the Guideline strongly recommended that patients receive breast-conserving surgery if they meet the indications. Meanwhile, SLNB was recommended as the first option for early breast cancer patients with $\mathrm{cN} 0$ lymph node staging $(8,9)$.

With the adjustment of China's fertility policy, the number of pregnant women has been increasing. Patients with suspected breast cancer during pregnancy should receive ultrasound-guided $\mathrm{CNB}$ to obtain a pathological diagnosis. Different treatment plans should be formulated according to the clinical stage, biological characteristics of the tumor, and gestational age of the patient. It is very important to implement individualized patient management plans with a focus on multi-disciplinary cooperation and the wishes of patients should be respected. The Guideline recommended modified radical mastectomy as the standard surgical procedure for PABCs. The timing of postoperative radiotherapy should be thoroughly considered before the patient receives breast-conserving surgery (10).

\section{Fundamental norms of surgical techniques}

Common basic surgical techniques of breast surgery include ultrasound-guided breast lesion biopsy, ultrasoundguided VABB, visualized percutaneous tissue marker clip placement, and central venous port implantation.

Ultrasound-guided breast lesion biopsy techniques include fine needle biopsy (FNB), CNB, and VABB, all of which have different characteristics and applications. Physicians should have a clear understanding of the indications of breast lesion biopsy, and choose the most appropriate biopsy method. The Guideline recommended $\mathrm{CNB}$ as the preferred biopsy method for breast lesions. This recommendation is based on level I evidence and the recommendation strength is class A. Meanwhile, since VABB can obtain more tissue samples, it may be used as a supplementary method for histopathological sampling (11).

In addition to diagnostic purposes, ultrasound-guided 
VABB can be used for resection of benign breast masses. The indications and contraindications of VABB should be strictly followed. For patients diagnosed with ADH, or borderline or malignant lobular neoplasms after VABB, open surgery was recommended for expanded excision (12).

The breast tissue marker clip is an imaging-visualized tissue marker which can precisely mark and locate the breast lesions and axillary metastatic lymph nodes with the assistance of ultrasound or X-ray. For unreachable suspicious lesions, use of marker clips is recommended to locate the lesion. The Guideline recommended that one clip should be placed in each breast lesion and each lymph node lesion with the assistance of ultrasound guidance (13).

With the development of the systemic treatment, a safe method for intravenous fluid access is generally required. The implantable central venous port has low local infection rates, low catheter displacement rates, and does not require dressing changes, making it a superior venous access device. The implantation site can be the internal jugular, subclavian, or basilic vein. Intravenous port implantation and/or maintenance should be performed by well-trained and qualified individuals and/or teams (14).

\section{Fundamental norms of surgical procedures for breast cancer}

Surgical procedures for breast cancer included SLNB, breast-conserving surgery, and modified radical mastectomy.

SLNB has become the preferred surgical procedure for staging axillary lymph nodes in breast cancer. Since Chinese breast surgeons are generally not qualified to use radioisotopes, and isosulfan blue is not readily available in China, the Guideline recommended carbon nanoparticles and methylene blue as the preferred tracers. This recommendation is supported by level I evidence and the has class A recommendation strength. Intraoperative frozen section pathological examination was recommended as the primary method for pathological evaluation in SLNB intraoperatively (15).

Modified radical mastectomy (the Auchincloss operation) is one of the most important surgical procedures in breast surgery in China. The Guideline provided specific recommendations for the operation position, the incision design, the layer and range of free skin flap, the tissues to be removed and retained during operations, surgical techniques, and the management of complications. Removal of level I and II lymph nodes in patients with metastatic lymph nodes was recommended (16).
Breast conserving surgery combined with whole-breast radiation therapy has been shown to be effective and safe in patients with early breast cancer. Patients in clinical stage I-II, with tumor size $\leq \mathrm{T} 2$, who are willing to conserve the breasts, and are able to retain a good breast shape post-operatively, are recommended for breast-conserving surgery. The Guideline recommended intraoperative frozen section pathological examination for margin evaluation. This recommendation is supported by level I evidence and has recommendation strength of class A (17).

\section{Genetic testing of breast cancer}

The importance of multigene profiling assays in adjuvant chemotherapy decision-making in early invasive breast cancer patients has been widely recognized. The Guideline emphasized the indications for multigene profiling assays, namely, T1-T2 breast cancer with positive estrogen receptor/progesterone receptor (ER/PR), negative human epidermal growth factor receptor 2 (HER-2), negative lymph nodes or limited metastases (1-3 metastatic lymph nodes), and clinically high-risk patients. The Guideline recommended detection of 70 genes (Mammaprint ${ }^{\circledR}$ ) or detection of 21 genes (Oncotype $\mathrm{DX}^{\circledR}$ ). These recommendations were support by level I evidence and class A recommendation strength, and level I evidence and class B recommendation strength, respectively $(18,19)$. To date, there have been few studies involving multigene testing in the Chinese population. Multi-center studies should be carried out as soon as possible to establish a national standard of multigene testing technology suitable for China.

The risk of breast cancer, ovarian cancer, and other diseases is significantly increased in women with BRCA1/2 germline mutations, and BRCA1/2 mutation carriers can reduce the risk of breast cancer through a series of interventions. Breast cancer patients with indications for BRCA1/2 testing and high-risk groups should receive professional oncogenetic counseling. The decision to accept or reject prophylactic mastectomy combined with reconstruction surgery should be made after considering the risks of disease, personal will, and the general condition of the patient (20).

\section{Prospects for new techniques in breast surgery}

In recent years, China has seen gradual developments in breast reconstruction technology after breast cancer surgery. This is conducive to helping female patients regain 
confidence and improve the patient's quality of life and that of their families. The Guideline emphasized the issue of professionalization in breast reconstruction surgery in China. Oncological safety must be adhered to, and R0 resection must be followed as a basic surgical principle, while not affecting standardized systematic treatments postsurgery.

Endoscopic surgical techniques have gained much attention from breast surgeons. To date, endoscopic nipplesparing mastectomy with or without immediate implantbased reconstruction surgery and endoscopic axillary or internal mammary lymph nodes dissection/SLNB has been actively explored in domestic clinical practice. Nevertheless, there is a lack of high-quality data on this technique and the Guideline has recommended future multicenter studies to obtain high-level evidence on endoscopic surgery in breast cancer.

\section{Conclusions}

As a professional academic society in the field of breast surgery in China, CSBrS always pays close attention to the basic clinical issues of breast surgery and is committed to strengthening multi-disciplinary cooperation and building an academic research platform that is suitable for China. The forthcoming Guideline is the first clinical practical guideline for breast surgery in China. The authors sincerely welcome comments and corrections from other experts, so as to improve the Guideline according to the latest research findings.

\section{Acknowledgments}

We acknowledgment $\mathrm{J}$. Teoh for the language editing of this article.

Funding: None.

\section{Footnote}

Conflicts of Interest: All authors have completed the ICMJE uniform disclosure form (available at https://dx.doi. org/10.21037/tbcr-21-8). YL serves as the unpaid editorial board member of Translational Breast Cancer Research from March 2020 to February 2022. The other authors have no conflicts of interest to declare.

Ethical Statement: The authors are accountable for all aspects of the work in ensuring that questions related to the accuracy or integrity of any part of the work are appropriately investigated and resolved.

Open Access Statement: This is an Open Access article distributed in accordance with the Creative Commons Attribution-NonCommercial-NoDerivs 4.0 International License (CC BY-NC-ND 4.0), which permits the noncommercial replication and distribution of the article with the strict proviso that no changes or edits are made and the original work is properly cited (including links to both the formal publication through the relevant DOI and the license). See: https://creativecommons.org/licenses/by-nc-nd/4.0/.

\section{References}

1. Sung H, Ferlay J, Siegel RL, et al. Global Cancer Statistics 2020: GLOBOCAN Estimates of Incidence and Mortality Worldwide for 36 Cancers in 185 Countries. CA Cancer J Clin 2021;71:209-49.

2. Guyatt G, Oxman AD, Akl EA, et al. GRADE guidelines: 1 . Introduction-GRADE evidence profiles and summary of findings tables. J Clin Epidemiol 2011;64:383-94.

3. Wang L, Liu L, Lou Z, et al. Risk prediction for breast Cancer in Han Chinese women based on a cause-specific Hazard model. BMC Cancer 2019;19:128.

4. Guidelines Committee of Chinese society of Clinical Oncology. Guidelines of Chinese Society of Clinical Oncology (CSCO) Breast Cancer. Beijing: People's Medical Publishing House, 2020.

5. Practice Bulletin No. 164 Summary: Diagnosis and Management of Benign Breast Disorders. Obstet Gynecol 2016;127:1181-3.

6. Rizzo M, Gabram S, Staley C, et al. Management of breast abscesses in nonlactating women. Am Surg 2010;76:292-5.

7. Peng Y, Xie F, Zhao Y, et al. Clinical practice guideline for breast fibroadenoma: Chinese Society of Breast Surgery (CSBrS) practice guideline 2021. Chin Med J (Engl) 2021;134:1014-6.

8. Jiang Z, Song E, Wang X, et al. Guidelines of Chinese Society of Clinical Oncology (CSCO) on Diagnosis and Treatment of Breast Cancer (2020 version). Transl Breast Cancer Res 2020;1:27.

9. Liu M, Wang CB, Xie F, et al. Clinical practice guidelines for diagnosis and treatment of invasive breast cancer: Chinese Society of Breast Surgery (CSBrS) practice guidelines 2021. Chin Med J (Engl) 2021;134:1009-13.

10. Loibl S, Han SN, von Minckwitz G, et al. Treatment of breast cancer during pregnancy: an observational study. 
Lancet Oncol 2012;13:887-96.

11. Ma JF, Chen LY, Wu SL, et al. Clinical practice guidelines for ultrasound-guided breast lesions and lymph nodes biopsy: Chinese society of breast surgery $(\mathrm{CSBrS})$ practice guidelines 2021. Chin Med J 2021;134:1393-5.

12. Li SJ, Hao XP, Hua B, et al. Chinese Society of Breast Surgery. Clinical practice guidelines for ultrasoundguided vacuum-assisted breast biopsy: Chinese Society of Breast Surgery practice guidelines 2021. Chin Med J 2021;134:1390-2.

13. Konen J, Murphy S, Berkman A, et al. Intraoperative Ultrasound Guidance With an Ultrasound-Visible Clip: A Practical and Cost-effective Option for Breast Cancer Localization. J Ultrasound Med 2020;39:911-7.

14. Sousa B, Furlanetto J, Hutka M, et al. Central venous access in oncology: ESMO Clinical Practice Guidelines. Ann Oncol 2015;26 Suppl 5:v152-68.

15. Ye JM, Guo BL, Liu Q, et al. Clinical practice guidelines for sentinel lymph node biopsy in patients with earlystage breast cancer: Chinese Society of Breast Surgery

doi: $10.21037 /$ tbcr-21-8

Cite this article as: Xiang H, Liu Q, Liu Y. Introduction to the key contents of the Chinese Society of Breast Surgery Practice Guideline 2021. Transl Breast Cancer Res 2021;2:18.
(CSBrS) practice guidelines 2021. Chin Med J (Engl) 2021;134:886-94.

16. Jiao DC, Zhu JJ, Qin L, et al. Clinical practice guidelines for modified radical mastectomy of breast cancer: Chinese Society of Breast Surgery (CSBrs) practice guidelines 2021. Chin Med J (Engl) 2021;134:895-7.

17. Chinese Association of Breast Surgery. A consensus statement on the breast-conserving surgery of earlystage breast cancer (2019). Zhonghua Wai Ke Za Zhi 2019;57:81-4.

18. Cardoso F, van't Veer LJ, Bogaerts J, et al. 70-Gene Signature as an Aid to Treatment Decisions in Early-Stage Breast Cancer. N Engl J Med 2016;375:717-29.

19. Sparano JA, Gray RJ, Makower DF, et al. Adjuvant Chemotherapy Guided by a 21-Gene Expression Assay in Breast Cancer. N Engl J Med 2018;379:111-21.

20. Genetic/Familial High-Risk Assessment: Breast, Ovarian, and Pancreatic Version 1.2021- September 8, 2020. Available online: http://www.NCCN.org 